\title{
Robota: Clever toy and educational tool
}

\author{
Aude Billard ${ }^{\mathrm{a}, \mathrm{b}, *}$ \\ a Autonomous Systems Laboratory, School of Engineering Sciences Techniques, \\ Swiss Federal Institute of Technology at Lausanne (EPFL), 1015 Lausanne, Switzerland \\ ${ }^{\mathrm{b}}$ Department of Computer Science, University of Southern California, 3641 Watt Way, Los Angeles, CA 90089-2520, USA
}

\begin{abstract}
Therapeutic and educational applications of robots have created a demand for robots showing a number of social skills. These skills include the capacity to imitate, to learn from demonstration, to interpret gestures and to recognize speech. Robot toys are an ideal platform to investigate the potential and limitations of human-robot social interactions.

This paper presents Robota, a mini-humanoid doll-shaped robot. Robota is used in an introductory robotics class at the undergraduate level. The class offers an introduction to different tools necessary for building human-robot social interactions. Through a series of hands-on projects, students learn how to use vision and speech processing and how to design learning algorithms. The goal of each project is to create an educational and entertaining game for normal and disabled children.

(c) 2003 Elsevier Science B.V. All rights reserved.
\end{abstract}

Keywords: Human-robot interaction; Educational toy; Humanoids; Social learning; Robotics class

\section{Introduction}

During the past 10 years, researchers have identified a number of social skills that robots ought to be provided with. These include the ability to recognize others, to interpret gestures and verbal expressions, and to recognize and to express emotions [5]. One of the most fundamental and, perhaps, the most difficult skill to program is the ability to learn from others. This is known as social learning in the study of natural systems [17]. Social learning includes learning by imitation, that is learning through the observation of others' behavior, and social facilitation, where the mere presence of others enhances learning of tasks.

\footnotetext{
* Tel.: +41-21-693-5464/+1-213-740-9223; fax: +41-21-693-7807/+1-213-740-5687. E-mail address: billard@usc.edu (A. Billard). URLs: http://www.asl.epfl.ch, http://www-clmc.usc.edu/ billard/
}

Teaching skills, whether cognitive, verbal or motors, is the very motor of human society, and relies importantly on human ability for social learning. Human ability to learn from socially interacting with others is so much taken for granted that the few people who are impaired in this ability (such as people with autism [7]) have enormous difficulty to adapt to society. Providing robots with the ability to learn from observing human behavior has, thus, become an important topic of research in robotics. The rational is that the robot's ability to imitate would offer a natural means of programming the robot, that would require none or minimal programming competence on the user's part. Robot learning by imitation, also known as robot programming through demonstration, finds its use for a large number of tasks, such as object manipulation $[13,16]$, learning body motions $[2,8,15]$, navigation $[5,10]$ and learning communication skills [4].

Robot toys are the most likely application of social robots in the near future [9]. Being cheap to build 
and likeable by a large part of the population, robot toys offer an ideal platform to test algorithms for social learning. Robota, a doll-shaped robot toy, was developed with the goal investigating what social skills would be required for a human-shaped robot to engage in believable social interactions [1]. Robota is currently being used in pilot studies with normal and cognitively impaired children, and with children with autism. The studies aim at developing Robota as a complementary educational tool that would guide the children through games that rehearse school material. In studies with autistic children, Robota is particularly interesting to educators as a means to systematically evaluate the child's social competences.

While the above-mentioned studies have been reported elsewhere $[3,6]$, the present paper focuses on the novel application of Robota as a laboratory platform in an introductory class to robotics taught at the undergraduate level. The class uses a set of 10 Robota robots. The class revolves around lab sessions during which the students learn to program the robot's micro-controller and the robot-PC interface, using both vision and speech synthesizing. The lab sessions aim at the development of games suitable for normal and disabled children.

Section 2 describes briefly the hardware of the Robota robots and the applications of Robota as a toy for children. Section 3 describes the application of Robota in an undergraduate robotics class. Sections 4 and 5 conclude this paper with a discussion of the choice of providing Robota with human-like features, vision and speech interfaces and how these can, in some cases, be important components for building social skills in a robot.

\section{The Robota robots}

Robota is a small humanoid robot, $45 \mathrm{~cm}$ high, with motors to drive its legs, arms and head. ${ }^{1}$ Robota electronics consists of 3 superimposed boards (PIC $16 \mathrm{~F} 8704 \mathrm{MHz}$, PIC $16 \mathrm{~F} 87016 \mathrm{MHz}$, and 68376 Motorola), that drive 6 motor outputs, 24 sensor entries (16 analog and 8 digital) and a set of sensors: 4 infra-red emitter/receivers, 2 light detectors, 2 py-

\footnotetext{
${ }^{1}$ A system to control 3-DOF movement of Robota's eyes is under development at the EPFL.
}

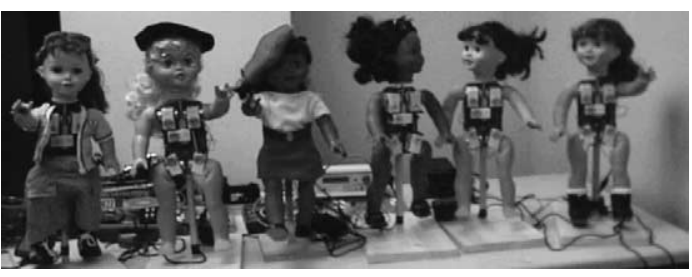

Fig. 1. Six Robota robots. From left to right: red-hair, blond-hair, Hispanic, African-American, dark-hair, light brown-hair Robotas.

roelectric sensors, 6 switches and 2 electro-magnets. Robota is interfaced to a PC or a PocketPC through an RS232 serial connection to use speech synthesis (ELAN), speech processing (Conversay, IBM Viavoice) and for processing video data from a QuickCam camera (CMOS, $640 \times 480,30 \mathrm{fr} / \mathrm{s})$. Programming of the robot is done in ANSI $\mathrm{C}$ and programming of the PC-robot interface is done in Visual $\mathrm{C}++$ or Windows CE (Pocket PC).

Robota's arms, legs and head are plastic components from commercial dolls, that are attached to Robota's main body. The doll parts have different skin colors and different facial features (African-American, Caucasian, Hispanic, etc.), ${ }^{2}$ see Fig. 1.

\subsection{Robota: the educational toy}

The social aspect of Robota lies foremost in its potential as an educational toy. The Robota robots are being used in different experiments that investigate the use of toy robots for normal children and for children with disabilities. These studies have been reported elsewhere, see $[3,6]$. Here is a brief summary.

Since 1998, tests have been conducted in children with autism, as part of the Aurora project, directed by Dr. Kerstin Dautenhahn, and in collaboration with the Colnbrook School and the Radlett Lodge School, a school of the British National Autistic Society. This research investigates how playing an imitation game with Robota can help autistic children learn to adopt body postures fundamental to social interactions [6]. In the imitation game, Robota mirrors the movements of the arm and of the head of the child, see Fig. 2.

\footnotetext{
${ }^{2}$ All Robota are female dolls simply because the toy market does not offer any boy doll with the required features $(30 \mathrm{~cm}$ height minimum and rigid body).
} 


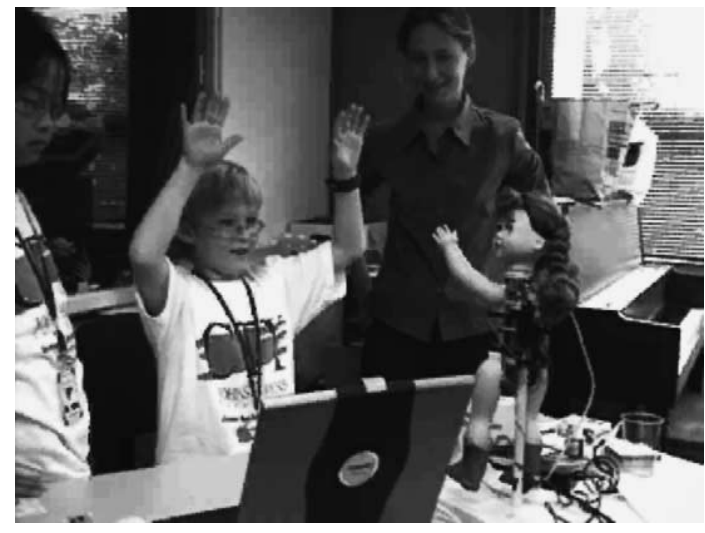

Fig. 2. A 10-year-old child learns about Robota vision-based imitation game, as part of the gifted children science and discovery summer program, Los Angeles, 26 June 2002.

The child must look straight at Robota's face for the imitation to occur. When the robot stops imitating, the child must initiate the interaction. Looking straight at someone else's face and initiating social interactions are less natural to children with autism than they are to normal children. The hope is that, once the child has become used to performing the body coordination required by the imitation game, the child will find it easier to perform the same body coordination when interacting with another child or an adult (as opposed to interacting with a robot).

The Aurora project conducts a comparative study using both a vehicle-like robot and Robota to evaluate the influence of using a robot with human characteristics (such as Robota). Body coordination games are as successful when using either Robota or the vehicle-like robot. However, it is easier to reenact some games played as part of the normal school curriculum with Robota than with the vehicle-like robot (especially games involving hand gestures and verbal dialog). This suggests that Robota might be a more suitable and more natural tool to achieve the desired transfer of learning of the from a child-robot context to a child-adult context.

Since October 2000, pilot studies have been conducted with children born premature (consequently suffering from motor deficits), in collaboration with Dr. Sharon Demuth at the USC Premature Infant Follow-Up Clinic. These studies test whether playing with Robota can be an incentive for the child to move her/his arms and legs (in order to let Robota dance) and, by so doing, to perform her/his daily motor exercises.

Robota's controller offers also a language game in which the robot can be taught a simple language, formed of a vocabulary and of regular syntactic rules, to express the robot's interactions with the teacher (e.g. "you move your left arm", "I touch your right foot", etc.). The language game and the imitation game are examples of artificial intelligence techniques (speech processing, visual processing and Hebbian learning applied to a feedforward neural network). These techniques and their implementation are sufficiently simple to be explained to normal children between 6 and 12 years old. For this reason, Robota's imitation game has being featured in on-line children's Internet guides (Finditquick, Weigl Educational Publishers, 2000, Bonus.com the SuperSite for Kids), and was presented at different science fairs, see Fig. 2. One Robota robot, offering the language game, is currently being displayed at the French national science museum, La Cité des Sciences et de l'Industrie in Paris (from November 2001 to July 2003).

\section{Robota course}

This section focuses on the latest application of Robota in an undergraduate introductory class to robotics, at the Computer Science department at the University of Southern California (USC). The class is entitled "Mechatronics: Programming Humanoid Robots". The class was given for the first time at USC during the spring semester 2001. It gathered the maximum allowed of 30 students: 28 males, 2 females. It is a 3-unit class. It will be given again in the 2003 fall semester, and then regularly once per year.

The Robota course emphasizes the applications of robotics and artificial intelligence techniques to facilitate human-robot interactions. It introduces some of the major issues behind computer vision, speech synthesizing and speech processing and trains the students to program some of these techniques to create interactive games with Robota.

The class format consists of lectures and laboratory sessions. The class uses a set of 10 Robota robots. The course work is based around the programming and 
Table 1

Syllabus of Robota CS class (each lecture lasts $1 \mathrm{~h}$ and $20 \mathrm{~min}$, each lab session lasts 3 hours)

- Introduction (two lectures): Biologically inspired and industrial robot applications. Robota applications.

- Motors (two lectures): Motors types and functioning. Construction of Robota motors.

1st assignment (1 week): Program Robota to dance for 2 minutes on the music of your choice.

- Sensors (two lectures): Sensor types and functioning: demo of Robota infra-red, switches, potentiometers, etc.

2nd assignment (1 week): Program Robota to react on pressure of the switches and motion of the potentiometers.

- Micro-controllers (two lectures): Description and programming of the three micro-controllers of Robota.

- Speech synthesis and processing (two lectures): Basic algorithms for speech processing and synthesis. Demo of ELAN synthesizers and Microsoft speech recognition software.

3rd assignment (2 weeks): Program Robota to interpret written text and to reply using Robota speech synthesizer.

- Video processing (four lectures): Introduction to key issues behind video processing. Demo of video processing code for the Robota camera.

4th assignment ( 3 weeks): Program Robota to imitate the user's arm and head motions by segmenting the optical flow from

Robota camera.

- Learning and control architectures: Applications of ANNs and GAs to robotics. Demos of Robota ANNs programs.

Final project (5 weeks): Create a robot toy. All projects must involve a learning component.

design of the Robota-PC interface and of the Robota micro-controller. The class is composed of lectures and hands-on practicals, see the syllabus in Table 1 . The practicals consist of a set of four assignments and one final project, which students accomplish in teams of two or three. Through the practicals, students are led to think about what it takes to create a social robot. They discuss the importance of the physical appearance of the robot, the limitations of the state-of-the-art vision and speech processing for recognizing human gestures and interpreting human speech. The course calls to the students' imagination and originality. Each team of students design its robot's controller and physical appearance, so as to create a unique educational and entertaining robot toy.

In their final project, teams are presented with a choice of projects. A common requirement to all projects is the implementation of visual segmentation or speech processing and the implementation of a learning technique presented in class. The outline of each project specifies an outcome related to the experiments with normal and disabled children. The remaining of this section describes three of these projects.

\subsection{Robota learns to dress}

Dressing-up is an everyday task which we practice without thinking, at least as far as the order in which we put clothes on is concerned: underwear first, then shirt, then trousers, etc. When we are cold or warm, we spontaneously put on or off a piece of clothing. All children have to acquire these routine motions one day and stop relying on mummy or daddy to tie their shoes and put on their jackets. Retarded children with autism have sometimes a hard time acquiring the logic and reflexes for getting dressed up. This is perhaps one of the most wearing tasks for the parents of these children, who find themselves repeating the same sentences and gestures over days and years. Last October, during one of the Aurora testing sessions, the mother of a 16-year-old child with autism expressed her wish to see Robota help reminding her child of the list of instructions to get dressed. This 16-year-old child was severely retarded and still relied on her mother's help to get dressed. The child loved dolls and used to carry one constantly with her. For this reason, her mother was confident that Robota, because of its resemblance to a normal doll, would be a close companion to her child and could be useful in participating actively in the daily routine of her child.

The outline of the class project Robota learns to dress aimed at developing a game that would fulfill this mother's request. In the game, the child/user dresses up Robota and, by doing so, is reminded of the logical routine of putting clothes on, see Fig. 3. The game uses color recognition and associative learning techniques seen in class. A video processing program allows Robota to distinguish between different pieces 


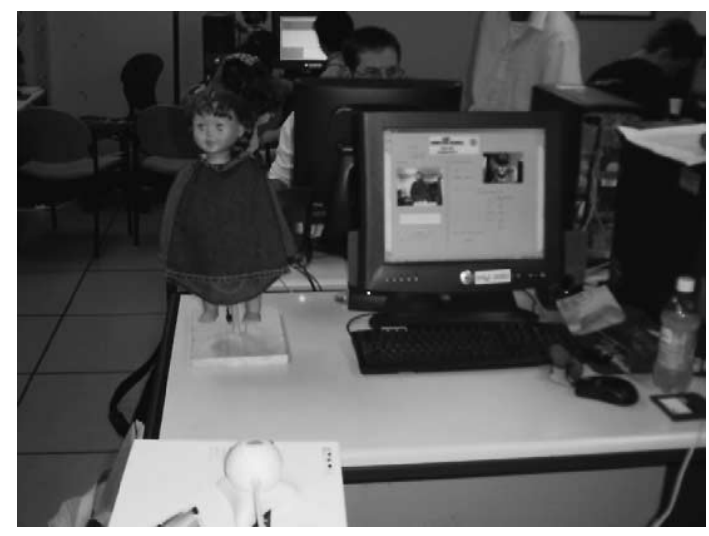

Fig. 3. The setup for Robota learns to dress up. Project by Alex Chan, Johnny Li and Siu Lun Wu. The camera is placed in front of Robota at a fixed distance of $60 \mathrm{~cm}$. The camera output is visible on the PC screen.

of clothing (e.g. trousers, shoes, jumper) by detecting their colors. Robota's potentiometers are used to detect that Robota is being dressed up, by measuring the passive motion applied on her limbs. Robota initiates the game by asking for each piece of clothing (e.g. Robota says "Put my shirt now"). Robota is able to tell when she is not given the correct piece of clothing: (e.g. Robota says, e.g. "Do not put my shoes now, you must first put my trousers on!"). When the game is switched into learning mode, Robota can be taught the correct order in which to put the clothes. Using an associative memory type of neural network, Robota associates specific motions with specific color tissues. The user can test that Robota has learned correctly by going into the testing mode. For example, when presenting a red T-shirt to Robota in the testing mode, Robota would move its arms up above its head so that its arms are more accessible.

\subsection{Robota drawing game}

Drawing is a major pre-school activity, much reinforced in pre-school and elementary school. A child's drawing reflects his/her interpretation of the relationship across things and beings, and is a mean for the child to develop a symbolic representation of his world. It also reflects the child's capability to control his arm, hand and fingers in a well-coordinated fashion. Children with motor and cognitive deficits

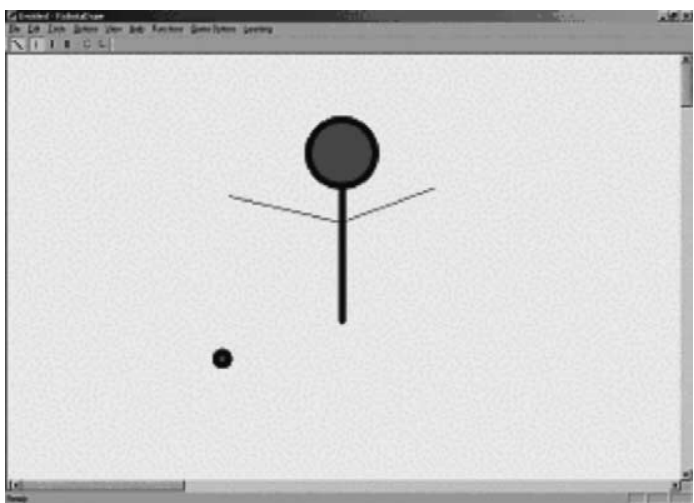

Fig. 4. Stick figure in the drawing application of the Robota Drawing Game.

have sometimes trouble controlling their arm muscles sufficiently well to draw. One psychological test, conducted on 2-year-old children, at the USC Premature Infant Follow-Up Clinic measures the child's degree of handicap, by asking the child to draw simple geometrical forms: straight lines, triangles, circles.

The outline of the project Robota Drawing Game aimed at developing a game that would provide an incentive for the child to draw straight lines, as well as measure the child's ability to do so. The game combines speech and learning techniques. In the game, Robota recognizes postures (sitting, lifting up a leg/arm) of a stick figure drawn by the child on a drawing pad or on a Touch screen, see Fig. 4. The game offers three levels of complexity. At the lowest level, Robota simply reproduces the posture of the stick figure in the drawing, by moving adequately its arms and legs. Robota guides the child by commenting on the correctness of the motion and by responding to the child's query.

Level two of the game is designed to increase the child's motor and coordination skills. This game starts by placing a random point on the screen that serves as a target for the child to reach with the pen. A successfully completed action implies to draw a straight line from the starting point to the target point. The start point is specified by the position of the target (e.g. if the target is in the lower left corner of the screen, the child should start from the region along the body line of the stick figure that corresponds to figure's right leg). When the action is successful, the robot responds 
by moving the limb to the position specified by the target point. The robot helps the child direct his arm to the correct starting point, through verbal comment "This is not my left leg!", and draw straight, e.g. saying "This is no longer straight. Move more to the left".

In level three of the game, a learning mechanism allows the user to teach Robota the correspondence between the stick figure and Robota's limbs. A Hebbian associative memory associates the position of Robota's limbs, returned by the potentiometers, with the position of the cursor on the screen. This level was designed to allow the educator to change at will the requirements of the levels 1 and 2 of the drawing task to test the child's ability to adapt to new visuo-motor coordination (e.g. swapping left-right motions).

\subsection{Robota counting game}

Learning to count is a fundamental stage of elementary schooling. For children with verbal and cognitive handicap, this requires long training and a lot of incentive. The outline of the Robota Counting Game project aimed at developing an interactive game to teach young children to perform additions. The game starts with Robota asking the child to count up to a number, randomly generated, e.g. Robota asks "what makes 3 plus 4 ?". If the number is smaller than 10 , the child must respond, by raising his arm the appropriate number of times. A visual tracking system detects motion of the child's left and right arm (lifting up the left arm corresponds to an increment of 1; lifting up the right arm specifies the end of the counting). Robota mirrors the child's arm motion (lifting up left and right arm with the child) to provide a feedback of the tracking system.

When the number is bigger than 10 , five potential answers (4 incorrect, 1 correct) appear on an image of Robota displayed on the PC. Each answer is correlated with a particular limb (for instance, in the above addition example, the display could show "7" on left arm, "10" on the right arm, 5 on the "head", etc.). The child answers Robota's question by pressing the switch on the limb he believes correspond to the correct answer (in the above example, the left arm). If the child does not know the answer, he can ask for a hint. Robota will then move two of its limbs indicating that the answer is one of these two limbs (in the above example, moving both left and right arms).
Robota congratulates the child once he has found the right answer, or encourages him if he makes a mistake. Robota keeps track of the child's mistakes and chooses the questions, such that the game tends over time to focus on the child's weak points.

\section{Discussion: key features required for social robots}

Work on Robota is driven by the aim of designing a robot that fulfills a number of social tasks: education, entertainment, therapy. For each of these tasks, the robot is provided with competences for performing a specific social interaction, which is specified by the rules of the game. Throughout the design of Robota, a number of decision have been made concerning the physical appearance and capabilities of the robot. This section reviews and discusses these decisions.

\subsection{Human-like features}

Opponents to humanoid robotics question whether robots ought to be physically similar to humans. The likely answer is "it depends on the task". If a robot is to learn from humans, it should, in some way, be capable to behave similarly to humans. For instance, in order to learn how to open a box, the robot must be capable of moving the lid in the same direction as that demonstrated by the human instructor. Opponents to anthropomorphic hands have argued that, for most manipulation tasks, having a human hand would not be more beneficial. Indeed, in well-defined industrial environments, robots are provided with grippers specifically designed for the task, that do not resemble the human hand or arm, but are more efficient that the human arm. There are tasks, however, for which human features are important. For instance, if the robot is to learn sign languages to communicate, it should be provided with something that closely resembles human hand's digits and have arms that provide the degrees of freedom required to reproduce the specific gestures.

In the case of the Robota robots, there was an important incentive to provide the robot with a number of human features, such as the legs, arms and head of a lovely looking baby-girl. Because Robota was meant to help children learn about specific social interactions 
(such as counting, dressing-up and imitating body motions), it was important to develop a robot human-like in its social capacities. Robota's human features set the stage for engaging in human-like types of interactions, that would not be naturally phantomed by the child if the child would be to play with an animal-like robot (e.g. the Furbys, SONY's AIBO) or with a vehicle-like robot (e.g. LEGO Mindstorms).

\subsubsection{Engineering projects: Robota skis and drives a car}

Two projects of the Robota class were particularly challenging in the engineering development they requested. While these two projects did not develop a social competence in Robota, they highlight the idea that a human-like body opens the door to a large variety of applications.

One project developed a Robota that does a kind of cross-country skiing. Robota is attached to a home-made pair of skis and can propel itself forward using sticks, see Fig. 5. Robota also responds to the presence of a green or red flag. The camera observes the area in front of Robota. When a green flag is present, Robota starts moving. When a red flag appears, Robota stops.

Another project got Robota to drive a remote control car, see Fig. 6. The motors of Robota's two legs were interconnected to the two motors of a remote control car. Switches were attached to the car to create a frontal bumper and connected to Robota's micro-controller.

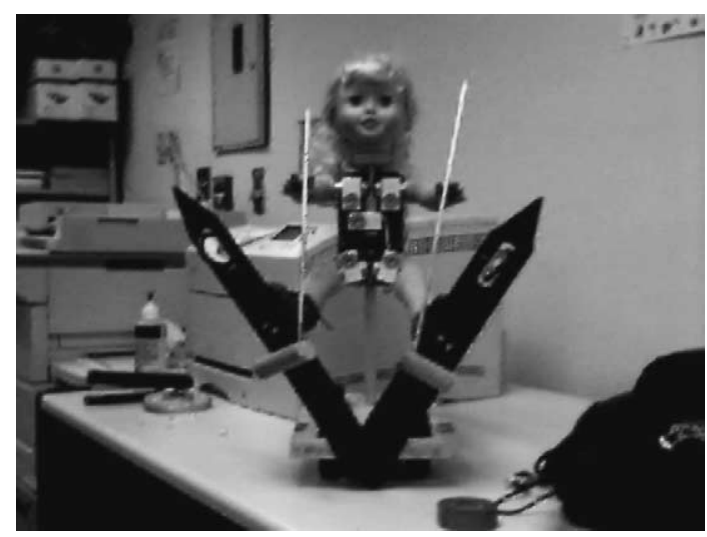

Fig. 5. Robota on skis. This is the "Oh no! I'm falling off the mountain!" pose. Project by Eric Grandma, Shannon Pierce and Max Gostomelskiy.

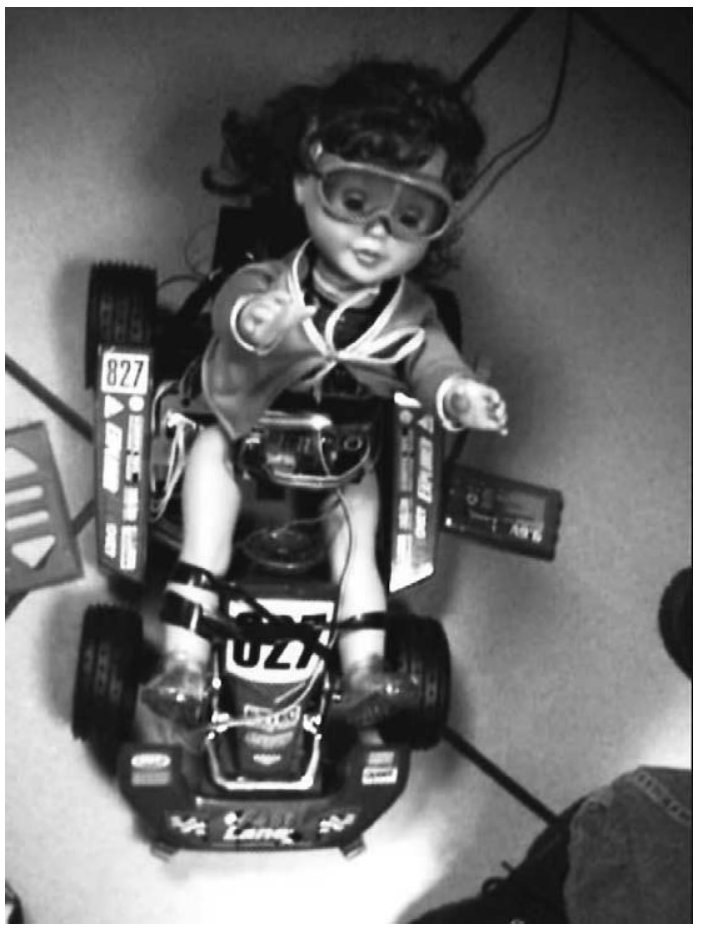

Fig. 6. Robota drives a car. Project by Amit Agrawalla, David Lam and Charles Staples.

Robota's micro-controller was, then, programmed, so that Robota could send commands to control the moving and turning motions of the car in reaction to the bumper signals.

\subsection{Visual processing}

Visual processing is a major component of human-robot interactions. It provides the information necessary for the robot to interpret, react to and learn from observing the behavior of a human instructor. Vision processing occupies a large part of computer science research. It remains, however, a very difficult and challenging research topic. Successful tracking of human motion still requires carefully monitored conditions (stable lighting, fixed distance person-camera). Nevertheless, machine vision has made important progress in the past decade, that will eventually lead to the development of systems allowing real time tracking of body motion in an everyday environment. 
The experiments with disabled children provided an important incentive to provide Robota with vision. Vision allowed us to remove the constraint of having the child wearing sets of active infra-red sensors that could track the child's hand and head motion during the imitation game. The vision-based imitation game makes it a more natural and less constraining setup for children with autism, who sometimes dislike wearing foreign objects.

Because vision plays a key role in human-robot interaction, it became an important teaching agenda of the Robota class. The class introduces key topics of visual processing, such as optical flow, color filtering and stereo vision, which students can then experiment through the assignments. For instance, in the fourth assignment of the class, see Table 1, students must develop a simple vision processing system that enables the robot to track upward movements of the user's hand and sideway shaking of the user's head. They, then, implement a simple imitation game, in which the robot replicates, in mirror fashion, the head and arm movements of the demonstrator, see Fig. 7. The

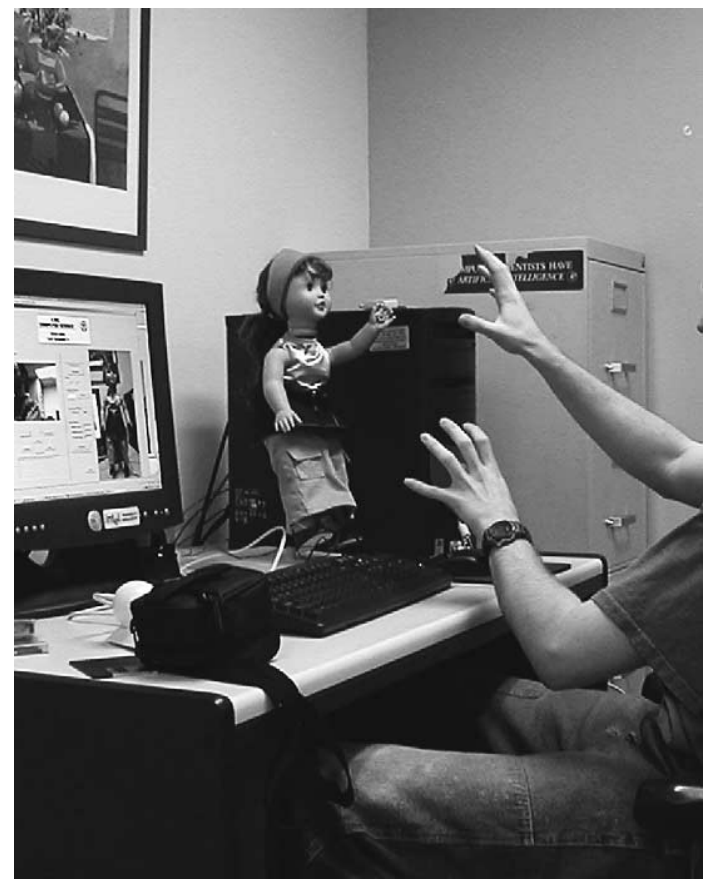

Fig. 7. Project Robota Counting Game by Adam Robert, Zach Turner and Dale Hersowitz. In the picture, Robota imitates Zach's arm movement and adds up after each. students have to work in an open laboratory in which the lighting and background conditions cannot be controlled and with a very noisy camera system (CMOS QuickCam). Nevertheless, during the class, all teams managed to develop a simple system that exploits optical flow and lighting intensity gradient, with a $90 \%$ efficiency.

\subsection{Speech processing}

Speech is another major component of human social interactions. It complements gesture-based interaction and opens the door to purely cognitive tasks. The study of speech processing and synthesizing is as important a research field as is machine vision. Similarly to vision, providing Robota with speech processing and synthesizing allowed us to remove the constraint of using a special equipment with the children. For instance, it allowed us to reimplement the language game without the need of using a keyboard. Typing on a keyboard is not convenient when testing children younger than 5 years old.

Because speech is an important tool for implementing human-robot interaction, speech processing became a second important topic of the Robota class. The class covers the basic principles of speech synthesizing and some key issues of Natural Language Processing (NLP). In the third class assignment, see Table 1, students implement a simple NLP interface for Robota. Robota must be capable of recognizing a number of key words and key phrases and of producing a sensible answer to any query. ${ }^{3}$ For this assignment, students were left with the freedom of choosing the topic of interest of the robot. As a results, students chose the robot's character in accordance to their own interest. They, then, dressed their robot accordingly to its character (grunge, easy-going, school-like, party-like) and programmed speech interaction to reflect this character. For example, one Robota was keen on talking about sport, while another one had lots to say about drinks and food (with often hilarious humor). A third Robota featured a little girl growing up in a farm and could talk about all animals living in that farm.

\footnotetext{
${ }^{3}$ Because of the noise level in the laboratory, students were not able to use Robota speech processing interface and, instead, used written text typed on the PC keyboard as the user input for the NLP program.
} 


\subsection{What does it take to actually build social robots?}

Building a robot capable of social learning, namely learning from the observation of human behavior, requires the integration of several disciplines, including machine vision, natural language processing and automation. Each of these disciplines are usually taught separately and seldom through hands-on practicals. The curriculum of the Robota class was developed with the goal to provide an introduction to some of the key components of programming social learning competences in a robot, such as speech processing and machine vision.

The recent development of end-users applications that exploit speech and visual processing have made available a large number of open-source utilitarians that make it easy for a developer to interface speech and visual input with a classical $\mathrm{C} / \mathrm{C}++$ application. Therefore, it is now conceivable to teach a robotic class that includes, but does not focus solely on, speech and visual processing. In the Robota class, the speech interfaces (ARL and TTS) and visual processing interface are treated as one of the many readily available modalities of Robota (as much as infra-reds, touch sensors and potentiometers). The major part of the teaching and programming effort focus on interfacing these modularities and on developing adaptive algorithms (artificial neural networks, genetic algorithms and reinforcement learning) to provide the robot with the capacity for social learning, i.e. to learn from interacting with the user.

Most hands-on robotic classes focus on the building of vehicle-like robots, sometimes enhanced with manipulators $[12,14]$. The programming part of such classes focus, then, on the design of control algorithms to achieve safe navigation and efficient object manipulation. The robot is usually designed with the goal to participate in a competitive game, involving, for instance, the removal of pucks or the pushing of balls in a soccer-like fashion. These classes have had a tremendous success in teaching principles of mechanical design and sensory-motor coupling. These are fundamental principles of robotics which gain at being experienced through hands-on classes. Similarly, higher levels concepts of control, involving artificial intelligence techniques for optimization and learning, would gain to be implement in real hardware setup. This has for long been hindered by the cost of electronic boards that would powerful enough to enable the real-time computation of video images and speech. Thanks to the recent mass commercialization of Pocket PCs (e.g. The Compaq iPAq, $250 \mathrm{MHz}, 32 \mathrm{Mb}$ RAM, costs around \$400), CMOS cameras (The Logitech QuickCam costs \$49) and speech engines (the license for IBM Viavoice ARL and TTS costs around \$200), building a basic robotic setup for performing simple visual and speech processing now costs less than $\$ 700$. This price lies within the range of that spent by most western universities for, e.g. one LEGO-based kit used in a hands-on robotics classes.

Consequently, a few universities have recently developed classes in which the focus is no longer on how to build the robot but on to design complex algorithm to control the robot and its many interfaces. The Khepera robots (K-Team SA) with their on-board 2D cameras and the pioneer robots (Active Media SA) with their sonars are both (increasingly) affordable teaching tool, that are fully functional once purchased, leaving the programming load to higher-level control algorithms. The Khepera robots have been used in learning systems classes (given by Dario Floreano at EPFL, Wolfgang Mass at the Technical University in Graz and Henrik Lund at University of Aarhus). The Pioneer robots have been used in a class on Kalman filters and Bayesian learning (Horswill at Northwestern University [11]). The Robota class was created in the same spirit as those classes. The 10 Robota robots used in the class were built by DIDEL SA (Lausanne, Switzerland), and came with a standalone low-level control system, leaving the freedom to concentrate on higher level of control.

\subsubsection{Note}

The Robota class curriculum and videos of the projects are publicly available through the class website, see http://www-clmc.usc.edu/billard. It is my hope that sequels of this class will be pursued in different universities. A simulator of the Robota robot has been developed by Cyberbotics SA (http://www.cyberbotics.com). A package comprising the class material, licenses of the Robota simulator 
and several Robota robots is available commercially from DIDEL SA (http://www.didel.com).

\section{Conclusion}

Vision and speech are key elements of social interactions. Vision provides the means to interpret context-specific behaviors, such as gestures. Speech offers the means to seek and transmit information not readily available visually, such as past and future facts, as well as to provide explicit teaching, such as category differentiations. This paper presented the application of speech processing and machine vision to develop an educational toy robot, Robota, that is being used in pilot studies with disabled children.

Developing new Engineering and Computer Science curriculum that emphasize the combined use of information technology and classical techniques from engineering is an important step toward the development of robots capable of complex interaction, involving gesture recognition and speech interpretation. Vision, speech processing, motor control and motor learning are usually taught in separate classes and are, seldom, presented in a common application.

This paper presented a new curriculum for an introductory robotics class at the undergraduate level, which offers an introduction to each of these fields through the design of a single common application: the creation of a highly interactive robot toy. While the class does not replace vision, speech processing and control theory classes, it offers students the chance to use concepts learned in these other classes for a specific application. Through the different projects of the class, students are led to think about what it is to provide a robot with human-like competences, whether these are purely motor (as in Robota drives a car or skies), or whether these are more cognitive (as in Robota teaches to count and learns to dress up). In all projects, the robot has to show social skills by reenacting a typical human-like social behavior. The class projects are driven by specific requirements for the game to be used with disabled children. The games developed in the spring class will be tested with children during the year 2003. Tests will be instructional in determining the validity of the games as educational tools for children.

\section{Acknowledgements}

The mechanical parts of the Robota robots were built by André Guignard, senior technician at the EPFL. The electronic parts of the Robota robots were created by DIDEL SA (http://www.didel.com). Some Robota robots also use the Kameleon board (K-Team SA). The equipment for the class (i.e. the Robota robots and PC's) was sponsored by an innovative teaching grant from the Intel Corporation to Aude Billard. The research projects conducted with Robota are supported by a Professor Boursier award to Aude Billard from the Swiss National Science Foundation.

\section{References}

[1] A. Billard, Drama, a connectionist architecture for on-line learning and control of autonomous robots: experiments on learning of a synthetic proto-language with a doll robot, Industrial Robot 26 (1) (1999) 59-66.

[2] A. Billard, Learning motor skills by imitation: a biologically inspired robotic model, Cybernetics Systems Journal 32 (1-2) (2001) 155-193 (Special Issue on Imitation in Animals and Artifacts).

[3] A. Billard, Play, dreams and imitation in Robota, in: K. Dautenhahn, B. Edmonds, L. Canamero (Eds.), Socially Intelligent Agents, Kluwer Academic Publishers, Dordrecht, 2001.

[4] A. Billard, Imitation: a means to enhance learning of a synthetic proto-language in an autonomous robot, in: $\mathrm{K}$. Dautenhahn, C. Nehaniv (Eds.), Imitation in Animals and Artifacts, MIT Press, Cambridge, MA, 2002.

[5] K. Dautenhahn, Getting to know each other-artificial social intelligence for autonomous robots, Robotics and Autonomous Systems 16 (1995) 333-356.

[6] K. Dautenhahn, A. Billard, Games children with autism can play with Robota, a humanoid robotic doll, in: S. Keates, P.M. Langdon, P.J. Clarkson, P. Robinson (Eds.), Proceedings of the First Cambridge Workshop on Universal Access and Assistive Technology [CWUAAT], Springer, London, 2002.

[7] G. Dawson, A. Adams, Imitation and social responsiveness in autistic children, Journal of Abnormal Child Psychology 12 (1984) 209-225.

[8] J. Demiris, G. Hayes, Imitation as a dual-route process featuring predictive and learning components: a biologicallyplausible computational model, in: K. Dautenhahn, C. Nehaniv (Eds.), Imitation in Animals and Artifacts, MIT Press, Cambridge, MA, 2002.

[9] A. Druin, B. Bederson, A. Boltman, A. Miura, D. KnottsCallahan, M. Platt, Children as our technology design partners, in: A. Druin (Ed.), The Design of Children's Technology, The Morgan Kaufmann Series in Interactive Technologies, Morgan Kaufmann, San Francisco, CA, 1998. 
[10] P. Gaussier, S. Moga, J.P. Banquet, M. Quoy, From perception-action loop to imitation processes: a bottom-up approach of learning by imitation, Applied Artificial Intelligence 7 (1) (1998) 701-729.

[11] I. Horswill, A laboratory course in behavior-based robotics, IEEE Intelligent Systems 15 (6) (2000) 16-21.

[12] J. Jones, A. Flynn, Mobile Robots: Inspiration to Implementation, A.K. Peters Limited, London, 1993.

[13] M.I. Kuniyoshi, I. Inoue, Learning by watching: extracting reusable task knowledge from visual observation of human performance, IEEE Transactions on Robotics and Automation 10 (6) (1994) 799-822.

[14] I. Nourbakhsh, Robotics and education in the classroom and in the museum: on the study of robots, and robots for study, in: Proceedings of the Workshop for Personal Robotics for Education, IEEE ICRA, 2000.

[15] B. Scassellati, Imitation and mechanisms of joint attention: a developmental structure for building social skills on a humanoid robot, in: C. Nehaniv (Ed.), Computation for Metaphors, Analogy, and Agents, Lecture Notes in Artificial Intelligence, vol. 1562, Springer, Berlin, 1999.

[16] S. Schaal, Learning from demonstration, Advances in Neural Information Processing Systems 9 (1997) 1040-1046.
[17] A. Whiten, R. Ham, On the nature and evolution of imitation in the animal kingdom: reappraisal of a century of research, Advances in the Study of Behaviour 21 (1992) 239-283.

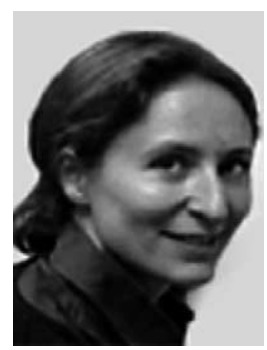

Aude Billard is SNF (Swiss National Science Foundation) Assistant Professor in the School of Engineering Sciences and Techniques at the Swiss Federal Institute of Technology/Lausanne and Adjunct Professor of Computer Science at the University of Southern California. She received her B.Sc. (1994) and M.Sc. (1995) in Physics from EPFL, with specialization in Particle Physics at the European Center for Nuclear Research (CERN). She received her M.Sc. in Knowledge-based Systems (1996) and her Ph.D. in Artificial Intelligence from the Department of Artificial Intelligence at the University of Edinburgh. She has worked as a Post-doctoral Fellow at IDSIA and LAMI (EPFL, 1998-1999), then as Research associate (1999-2000) and as Research Assistant Professor (2000-2002) in the Computer Science Department at the University of Southern California. 